\title{
Similar phenomena at different scales: black holes, the Sun, $\gamma$-ray bursts, supernovae, galaxies and galaxy clusters
}

\author{
Shuang Nan Zhang ${ }^{1,2}$ \\ ${ }^{1}$ Physics Department and Center for Astrophysics, Tsinghua University, \\ Beijing, 100084, China \\ email: zhangsn@tsinghua.edu.cn \\ ${ }^{2}$ Key Laboratory of Particle Astrophysics, Institute of High Energy Physics, \\ Chinese Academy of Sciences, Beijing, China
}

\begin{abstract}
Many similar phenomena occur in astrophysical systems with spatial and mass scales different by many orders of magnitudes. For examples, collimated outflows are produced from the Sun, proto-stellar systems, gamma-ray bursts, neutron star and black hole X-ray binaries, and supermassive black holes; various kinds of flares occur from the Sun, stellar coronae, X-ray binaries and active galactic nuclei; shocks and particle acceleration exist in supernova remnants, gamma-ray bursts, clusters of galaxies, etc. In this report I summarize briefly these phenomena and possible physical mechanisms responsible for them. I emphasize the importance of using the Sun as an astrophysical laboratory in studying these physical processes, especially the roles magnetic fields play in them; it is quite likely that magnetic activities dominate the fundamental physical processes in all of these systems.

As a case study, I show that X-ray lightcurves from solar flares, black hole binaries and gammaray bursts exhibit a common scaling law of non-linear dynamical properties, over a dynamical range of several orders of magnitudes in intensities, implying that many basic X-ray emission nodes or elements are inter-connected over multi-scales. A future high timing and imaging resolution solar X-ray instrument, aimed at isolating and resolving the fundamental elements of solar X-ray lightcurves, may shed new lights onto the fundamental physical mechanisms, which are common in astrophysical systems with vastly different mass and spatial scales. Using the Sun as an astrophysical laboratory, "Applied Solar Astrophysics" will deepen our understanding of many important astrophysical problems.
\end{abstract}

Keywords. Sun: flares, Sun: corona, Sun: X-rays, X-rays: binaries: individual (Cygnus X-1), accretion disks, magnetic fields, gamma rays: bursts: individual (GRB 940217), supernovae: individual (SN 1987A), galaxies: active: individual (M 87), black hole physics

\section{Introduction}

To start with, I first categorize research methods in astronomy into four classes:

- Objects oriented: focus on studying particular types of objects, such as stars, galaxies, or clusters of galaxies, etc. Astronomers doing this type of research are normally called observers or observational astronomers;

- Physical processes oriented: apply known physical processes in order to explain all observed phenomena. Those doing such research are called theorists or theoretical astrophysicists.

- Data analysis/mining: apply new or existing data analysis methods to large quantities of data, in order to discover new phenomena. We call them data analysts. 
- "Similar phenomena at different scales": look for similar phenomena from astrophysical systems with very different scales, in order to find common physical mechanisms operating in them. Perhaps they can be called "Observational Physicists".

The last class is the focus of this review article. This method may be appreciated from another perspective. According to the well-known astronomy ladder of J.J. Drake, the "fun" of astronomical subjects going down in this order: cosmology/black holes, quasars/AGN, planet hunting, galaxies, Milky Way/CVs, stars and the Sun. On the other hand, the amount or details of information astronomers can collect go almost in the opposite order. Clearly as the Sun is a star in our backyard, it is one of the most accessible astronomical objects. The Sun is the only object we can collect rather direct information on its core, radiative zone, convective zone, photosphere, chromosphere, corona and its winds. Interestingly, it has been gradually realized that many astrophysical phenomena occurring in the Sun also take place in many other astrophysical objects with enormously different scales. It is thus very likely that similar physical processes are operating in all these different systems. We therefore can use the Sun as our closest astrophysical laboratory for probing many physical processes and then apply the knowledge to understanding other astrophysical systems. Perhaps we can call this research discipline "Applied Solar Astrophysics".

The remaining part of this report is divided into four sections. In section 2, I will show some similar phenomena between the atmospheres of the Sun and that in accreting black hole systems, between jets produced in all kinds of different astrophysical objects with vastly different scales, and between the triple-ring structures in a young supernova remnant and in a galaxy cluster centered at an active galaxy. Magnetic activities seem to dominate or at least greatly influence all these phenomena. In section 3, I will focus on a particular kind of similar phenomenon, i.e., the non-linear dynamics of X-ray variations between that of the Sun, of a stellar mass black hole binary and of a gamma-ray burst. Their X-ray variations show remarkably consistent properties which can be modelled by multiplicative non-linear dynamical processes, suggesting that their X-ray emission regions are made of many inter-connected nodes or elements; multi-scale magnetic field topology most likely plays a key role in the non-linear dynamics observed in all of them. In section 4, I will make some concluding remarks on using the Sun as an astrophysical laboratory to understand these similar phenomena at different scales, and suggest a future $\mathrm{X}$-ray instrument required to solve some existing open issues, in order to make further progress in "Applied Solar Astrophysics".

\section{Common magnetic activities dominating processes}

In this section, I review briefly three types of similar astrophysical phenomena over a huge range of astrophysical scales. Observational and theoretical studies indicate that these phenomena are all related to magnetic processes in which magnetic topology and energy release play fundamental roles.

\subsection{Similar atmospheric structures between the Sun and black hole systems}

The temperature inside the Sun decreases from about 15 million degrees in its nucleus, where thermal nuclear fusion produces about $99 \%$ of the Sun's radiation energy, to about six thousand degrees on its photosphere. However starting from its photosphere its atmospheric temperature increases to about ten thousand degrees in its chromosphere and to above one million degrees in its corona. Because the Sun's interior remains as the only energy source, this temperature inversion means that the solar atmosphere is not in an equilibrium with its interior thermal energy source. Such a temperature inversion, i.e., 

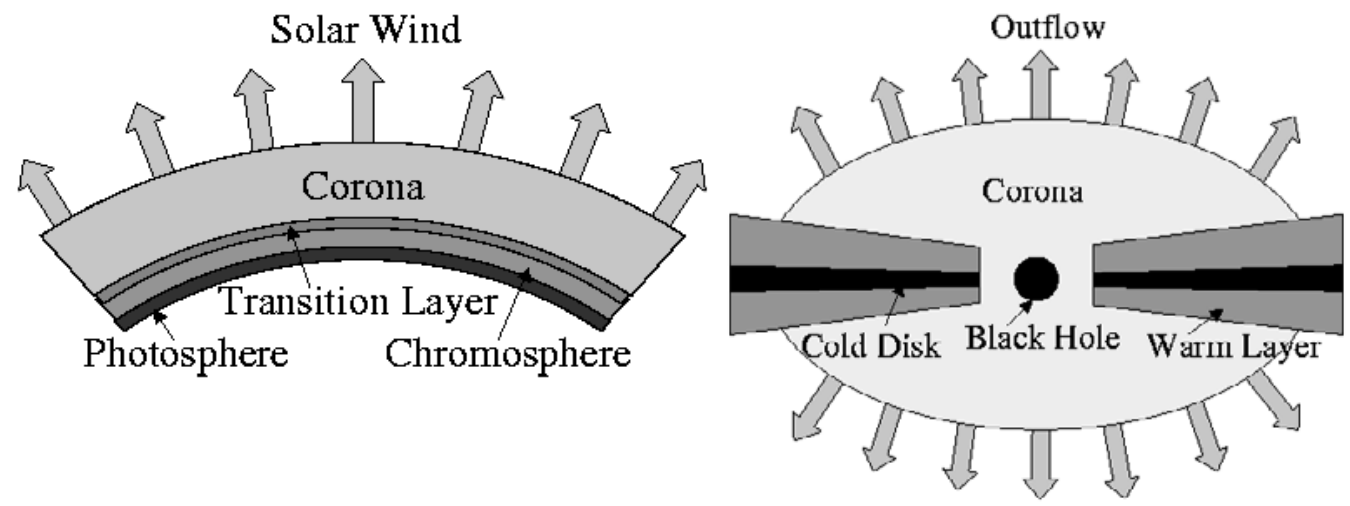

Figure 1. Schematic diagrams of the solar atmosphere and accretion disk structure. The temperatures in the solar atmosphere are approximately: $6 \times 10^{3} \mathrm{~K}$ (photosphere), $3 \times 10^{4} \mathrm{~K}$ (chromosphere), and $2 \times 10^{6} \mathrm{~K}$ (corona), respectively. For the black hole disk atmosphere, the corresponding temperatures are approximately 500 times higher: $3 \times 10^{6} \mathrm{~K}$ (cold disk), $1.5 \times 10^{7} \mathrm{~K}$ (warm layer), and $1 \times 10^{9} \mathrm{~K}$ (corona), respectively. (Re-produced from Zhang et al. 2000).

the solar corona heating problem still remains one of the most important problems in astrophysics. It is widely believed that solar flares driven by magnetic reconnections may be able to provide the required energy source for heating the solar corona, if sufficiently frequent 'nano'-flares (about $10^{24} \mathrm{erg}$ energy per event) are produced. This is the so-called Parker's conjecture (Parker 1983, Parker 1988, Parker 1991). However up to now searches for these speculated 'nano'-flares still remain inconclusive (Walsh 2003). Theoretically it also remains uncertain if it is physically possible to produce enough 'nano'-flares (Klimchuk 2006), even within the most hopeful avalanche models (Charbonneau et al. 2001). On the other hand, alternative models of solar corona heating may involve ion cyclotron waves and turbulence (see, e.g., Markovskii \& Hollweg 2004, Cranmer \& van Ballegooijen 2005).

The accretion disk around a black hole in an X-ray binary system has a similar structure to solar atmosphere (Liang \& Price 1977) and in particular the temperature inversion from its disk surface to its corona resembles that of the Sun, as shown in Fig. 1 (Zhang et al. 2000). Strikingly, the temperatures of the three regions in an accretion disk around a black hole are higher by approximately a factor of 500 than the corresponding regions in the Sun. This supports the notion that magnetic activity is responsible for powering the upper atmosphere in both cases, giving $T \propto E^{1 / 4} \propto B^{1 / 2}$ and thus $T_{\text {disk }} / T_{\text {sun }} \approx\left(B_{\text {disk }} / B_{\text {sun }}\right)^{1 / 2} \approx\left(10^{8} \mathrm{G} / 500 \mathrm{G}\right)^{1 / 2} \approx 500$, where the typical magnetic field strengths of $\sim 10^{8} \mathrm{G}$ for inner regions of black hole accretion disk (assuming equipartition) and $\sim 500 \mathrm{G}$ for the active regions of the Sun are used.

Since in an ionized accretion disk the angular momentum transport as well as energy dissipation are likely dominated by the so-called magneto-rotational instability mechanism (Hawley et al. 1999), X-ray flares or 'shots', driven by magnetic reconnection, may be responsible for the observed X-ray variability in systems ranging from accreting neutron star and black hole binaries, as well as active galactic nuclei (AGN) harboring supermassive black holes. Such a 'flare' model for accretion disks around black holes thus makes two generic predictions: (1) The X-ray emission region, including the corona in both stellar mass and supermassive black hole systems should have a 'disk-like' geometry, rather than a spherical-like geometry, which has been widely assumed; (2) Naturally their X-ray light curves should be made of individual flares or 'shots' if sufficiently good sensitivity and time resolution are available. 
Kubota et al. 2001 have identified a 'disk-like' configuration for the hot corona producing a hard X-ray power-law component through inverse Comptonization process, in the stellar mass black hole binary GRO J1655-40, which was discovered by Zhang et al. 1994 and in fact the second microquasar (X-ray binaries with superluminal jets) in the Milky Way (Tingay et al. 1995, Harmon et al. 1995. The X-ray emitting coronae in AGNs are suggested to have a 'disk-like' geometry, in order to explain the decrease of fraction of Type II AGNs as a function of the observed apparent X-ray luminosity (e.g., Ueda et al. 2003), due to the smaller projected area of the 'disk-like' corona for Type II AGNs (Zhang 2005, Liu, Zhang \& Zhang 2007). Flares or 'shots' are commonly observed from accreting systems of different sizes (Terrell 1972, Doi 1978, Miyamoto et al. 1988, Haardt et al. 1997, Nayakshin \& Melia 1997, di Matteo et al. 1999, Poutanen \& Fabian 1999, Negoro et al. 2001, Wang et al. 2004, Liu \& Li 2004). Modelling of periodic or quasi-periodic X-ray and infrared flares from Sgr A*, the supermassive black hole in the center of the Milky Way, suggests that these flares are of accretion disk origin (Aschenbach et al. 2004). It is thus quite possible that these flares are also produced by processes driven by magnetic reconnections.

We note that a hard X-ray power-law component up to at least 50-100 keV have also been seen from from active galactic nuclei (Bassani et al. 1996) and weakly magnetized (with surface magnetic field strength of around $\sim 10^{8} \mathrm{G}$ ) neutron star X-ray binaries (e.g., Zhang et al. 1996, Zhang 1997, Chen, Zhang \& Ding 2006), which often exhibit similar spectral state transitions as black hole binaries (Zhang 1997, Zhang et al. 1997a). It is well understood that the standard optically thick and geometrically thin accretion disk models (Shakura \& Sunyaev 1973) cannot produce this power-law component. However the orbital kinetic energies of protons in the inner accretion disk regions in weakly magnetized neutron star X-ray binaries, stellar mass black hole binaries and supermassive black hole systems should be approximately the same. Therefore, if the energy source of the above mentioned 'flare' model for accretion disks is dominated by particle's kinetic energies, the similar hard X-ray power-law component may be generated naturally. We comment in passing that the solar magnetic fields are also believed to be generated by dynamo mechanisms due to differential rotation in the Sun.

\subsection{Astrophysical jets at different scales}

Collimated outflows or jets are common astrophysical phenomena, now found in the Sun, proto-stellar systems, isolated neutron stars, neutron star and black hole binaries, gamma-ray bursts and supermassive black holes. MHD simulations have shown that differential rotation and twisted magnetic fields are two generic ingredients for generating collimated outflows (Meier et al. 2001), somewhat similar to magnetic reconnection processes in which twist and shear of magnetic flux tubes play very important roles for solar flares and perhaps also coronal mass ejection events (see, e.g., Wang et al. 2001, Pevtsov 2002, Yamamoto et al. 2005). With these two generic ingredients, their different scales and observational appearances (such as Lorentz factor and degree of collimation) of different astrophysical jets may reflect their different astrophysical environments (such as gravitational potential, differential rotation energy, magnetic field strength and topology, spin of the central object, etc), where jets are produced, accelerated, collimated and transported. Therefore, astrophysical jets can be used as powerful astrophysical probes for many different types of objects. For example, prolonged optical and X-ray afterglow emissions from gamma-ray bursts have been used to probe the properties of interstellar media around the gamma-ray burst progenitors and consequently the nature of their progenitors, because gamma-ray burst afterglow emissions are believed to be produced from external shocks when their jets eventually plow into their surrounding media, in a similar 

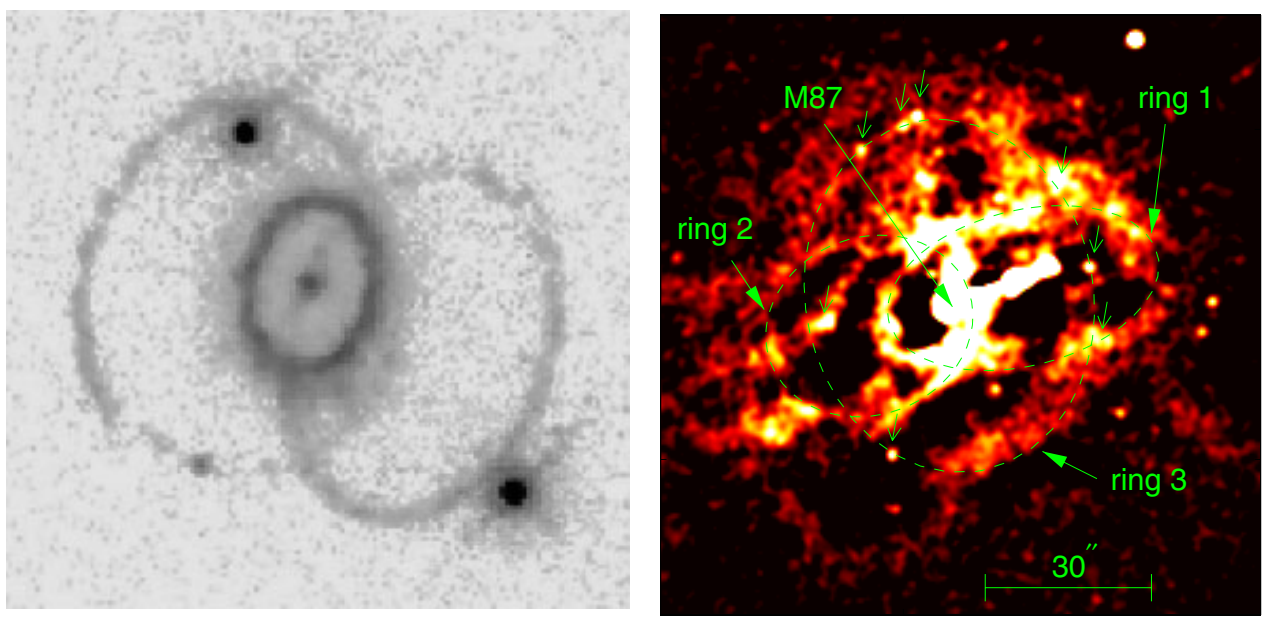

Figure 2. Similar triple-ring structures between the supernova remnant of SN 1987A and Virgo galaxy cluster centered at the active galaxy M 87. Left: The optical triple-ring structure of SN 1987A, as observed with Hubble Space Telescope (Burrows 1995). Right: The X-ray triple-ring structure of Virgo galaxy cluster as observed with the Chandra X-ray observatory (Feng et al. 2004).

way to an expanding supernova shell plowing into its surrounding medium. Interestingly, the same external shock model has been used to explain the decelerated relativistic jet from a black hole binary system (Wang, Dai \& Lu 2003), implying a dense surrounding medium and thus a massive star as the progenitor of the stellar mass black hole, similar to the progenitors to many long duration gamma-ray bursts (see, e.g., Meszaros 2001 for a review and references therein).

In many cases jets from black hole binaries, gamma-ray bursts and supermassive black holes are relativistic and may share the same production mechanisms, and it is very likely that black hole's spin energy is extracted to power their relativistic jets (see, e.g., Zhang et al. 1997c, Mirabel \& Rodriguez 1998, Mirabel \& Rodriguez 2002 for reviews and references there in). Using the X-ray continuum spectra, Zhang, Cui \& Chen (1997) first determined the spin of the black holes in several black hole binaries with relativistic jets and established the connection between black hole spin and jet observationally. This method of determining black hole's spin has since been further refined and widely applied to many more black hole binaries (for a recent review, see, e.g., Remillard \& McClintock 2006); the existence of extremally spinning black hole is now well-established.

\subsection{Similar triple-ring structures between a young supernova remnant and a galaxy cluster}

Another intriguing pattern of similarity is between the supernova remnant of SN 1987A and M87, an active galaxy producing a large scale jet in the center of the Virgo Cluster (Fig. 2). The triple-ring structure of SN 1987A observed in visible band with the Hubble Space Telescope is perhaps the most beautiful astronomical picture taken so far (see Fig. 2 (left); Burrows 1995). A MHD simulation by Tanaka \& Washimi (2002) showed that if its progenitor has similar large-scale magnetic field structure like the Sun, then its winds, similar to solar winds, will follow the twisted magnetic field topology and are thus preferentially located along its spin axis or in the equatorial plane. Suppose its progenitor has experienced a slow-wind phase (red-giant) and then a fast-wind phase (blue-giant), 
then the fast winds will eventually catch the slow winds to form the triple-ring like structure. It is possible that a triple-ring structure might be generic in a rotating system involving magnetized faster winds catching up slower winds. Meanwhile a central source of radiation is required for the rings to shine. For SN 1987A, the strong UV radiation from the supernova provides the necessary illumination.

In Fig. 2 (right) a triple-ring like structure in the X-ray band similar to that of SN1998A was shown, observed with the Chandra X-ray observatory (Feng et al. 2004). The ring sizes in the Virgo cluster are several thousand times larger than those of SN 1987A. It is possible that a catastrophic merging event around the M 87 nucleus is responsible for the triple-ring structure revealed here. In this scenario, a "slower wind" was present before the merging begins, e.g., a mixture of galactic winds from two merging galaxies. A "faster wind" was then driven during the merging process. The final merging of the two supermassive black holes releases a huge amount of high energy radiation to shine the triple rings in X-ray bands. Presumably, the resulting supermassive black hole spins rapidly to power the highly collimated M 87 jets.

\section{Non-linear dynamical processes at different scales}

In an extensive study of X-ray variability from a prototype black hole X-ray binary Cygnus-1 (Margon et al. 1973), Uttley et al. 2005 have shown that its X-ray flux fluctuations, characterized by rms (root-mean-squares) of its flux, is proportional to its mean flux. Such a flux-rms linearity cannot be modelled by additive or deterministic processes. Instead, Uttley et al. 2005 have demonstrated that the dynamical process for the X-ray production should be a non-linear 'multiplicative' process, which can be modelled by the following non-linear time-series model,

$$
\begin{aligned}
X_{i}= & 1+\sum_{j=0}^{\infty} G_{j} u_{i-j}+\sum_{j=0}^{\infty} \sum_{k=0}^{\infty} G_{j k} u_{i-j} u_{i-k} \\
& +\sum_{j=0}^{\infty} \sum_{k=0}^{\infty} \sum_{l=0}^{\infty} G_{j k l} u_{i-j} u_{i-k} u_{i-l}+\cdots
\end{aligned}
$$

where the coefficients $G_{j}, G_{j k}, G_{j k l} \ldots$ and the higher-order co-efficients are non-zero. Therefore the flux-rms linearity is a clear indication of non-linear dynamical process. Combining this and the fact that the flux distribution of Cygnus X-1 follows a lognormal distribution, but the peak flux distribution of solar flares follows approximately a power-law distribution (which is predicted by the self-organized criticality model), Uttley et al. 2005 thus claimed to have rejected essentially all previous models for $\mathrm{X}$-ray variability from X-ray binaries and active galactic nuclei, including shot noise, self-organized criticality, or dynamical chaos models (Uttley et al. 2005).

Since the characteristic X-ray variability seen in Cygnus X-1 has also been seen from other black hole X-ray binaries (Uttley \& McHardy 2001), as well as neutron star (Uttley 2004) and supermassive black hole accreting systems (Edelson et al. 2002, Vaughan et al. 2003a, Vaughan et al. 2003b), it has been suggested by Uttley et al. (2005) that the $\mathrm{X}$-ray variability in these systems should be driven by fluctuating accretion flow (Kotov 2001, Lyubarskii 1997). Since accretion flow does not exist in the Sun, their conclusion rules out common physical mechanism for X-ray emission from the Sun, thus has farreaching impacts to our understanding of some fundamental processes in a very broad range of astrophysical systems. 
Motivated by this and in light of the outstanding solar corona heating problem, we examine the X-ray variability in the Sun's X-ray lightcurves collected by the Reuven Ramaty High Energy Solar Spectroscopic Imager (RHESSI) (Lin et al. 2002)†, which has been in productive operation since launch on 5 February 2002 (Dennis et al. 2005). We also re-examine the non-linear dynamical properties of Cygnus X-1 and a gammaray burst. We demonstrate that a simple model can describe the non-linear dynamical properties of these three kinds of systems of very different astrophysical objects at very different scales.

\subsection{Flux-rms relation and flux distributions for individual solar X-ray flares}

In Fig. 3 the lightcurve, rms evolution and flux-rms correlation for RHESSI 2022619 solar flare are shown. The flux-rms relationship shown in the inset of Fig. 3 can be described by a simple model (plotted as solid line),

$$
\mathrm{rms}^{2}=(\alpha C)^{2}+C
$$

where $\alpha=0.27$ is a constant, $C$ is the average counts within each $1 \mathrm{~s}$ interval. Clearly, the total rms observed is made of two terms, a term linearly proportional to the average flux of the solar flux at a given time (plotted as dashed line), and a Poisson fluctuation term (plotted as dotted line); for a random Poisson process, $\mathrm{rms}=\sqrt{C}$. It can be seen from Fig. 3 that the linear term starts to dominate at flux above 20 counts per $0.1 \mathrm{~s}$.

In Fig. 4 and Fig. 5, eight RHESSI solar flares with different peak fluxes, durations and lightcurve morphologies are shown to illustrate their common properties. Interestingly, not only can the same simple model in equation (3.2) describes their flux-rms relation perfectly, but also $\alpha=0.27$ for all of them. In the insets the flux distribution of each solar flare is also plotted, which can be approximated by one or several log-normal components, i.e., the logarithm of the flux follows normal distributions, in comparison for the single log-normal peak of flux distribution for the black hole X-ray binary Cygnus X-1 (Uttley et al. 2005), and see the inset of Fig. 8 in section 3.3.

\subsection{Flux-rms relation for continuous solar X-ray light curves}

In Figs. 6 \& 7 several continuous segments of characteristic RHESSI solar X-ray lightcurves are shown in $3-6 \mathrm{keV}$ and $6-12 \mathrm{keV}$ bands with $1 \mathrm{~s}$ time resolution. These figures reveal $\mathrm{X}$-ray variability at all time scales with a large range of amplitudes over about five orders of magnitudes. Despite of its apparently very different lightcurves observed at well separated times, the solar X-ray variability exhibits remarkably consistent and simple relationship between the rms and its mean flux as shown in Fig. 8, which are again modelled by equation (3.2) with the same value of $\alpha=0.27$. We note that the flux-rms linearity also exists in a very broad frequency range if calculated from the FFT power-spectra of its lightcurves (Tang \& Zhang 2007), again similar to that of Cygnus X-1 (Uttley et al. 2005). It should be noted that a continuous solar X-ray lightcurve is collected over the whole surface of the Sun, not just from a single active region of the solar surface as for the case of a single solar flare. According to the mathematical description of equation (3.1) for the linear flux-rms relationship, this implies that many active regions in the solar surface are not isolated, but somehow connected and react to common triggers or ignitions. This is because the flux fluctuations from many isolated or independent regions should be proportional to the square-root of the total combined flux. Such inferred interconnection between different active regions may offer deep insight for the solar activity mechanisms.

$\dagger$ http://hesperia.gsfc.nasa.gov/hessi/ 


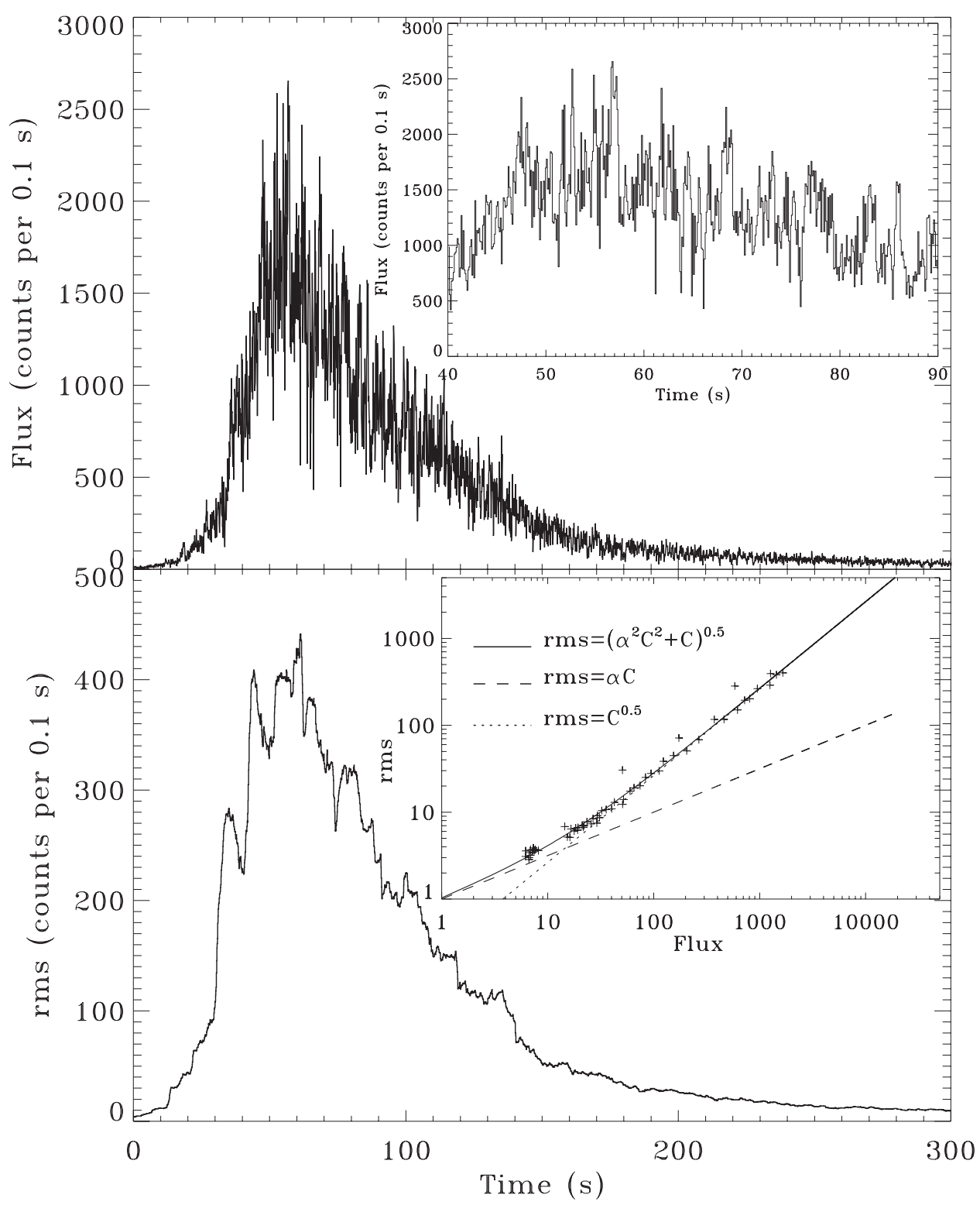

Figure 3. RHESS 2022619 solar X-ray flare between 3-50 keV. The upper panel and inset show its lightcurve at 0.1 second resolution; variability beyond Poisson counting fluctuations is clearly visible. The lower panel shows the rms (root-mean-squares), in units of counts in $0.1 \mathrm{sec}$, for each $1 \mathrm{sec}$ internal as a function of time and the average flux, in units of counts per $0.1 \mathrm{sec}$, during each $1 \mathrm{sec}$ interval. For the model lines shown in the inset, $\alpha=0.27$ is the best fit result with negligible error.

\subsection{Cygnus $X-1$ and a $\gamma$-ray burst}

In Fig. 9 the flux-rms correlation for the canonical stellar mass black hole binary Cygnus X1, observed with the PCA instrument onboard the Rossi X-ray Timing Explorer†, is shown (see Uttley et al. 2005 for comprehensive results on the nonlinear behaviours of 

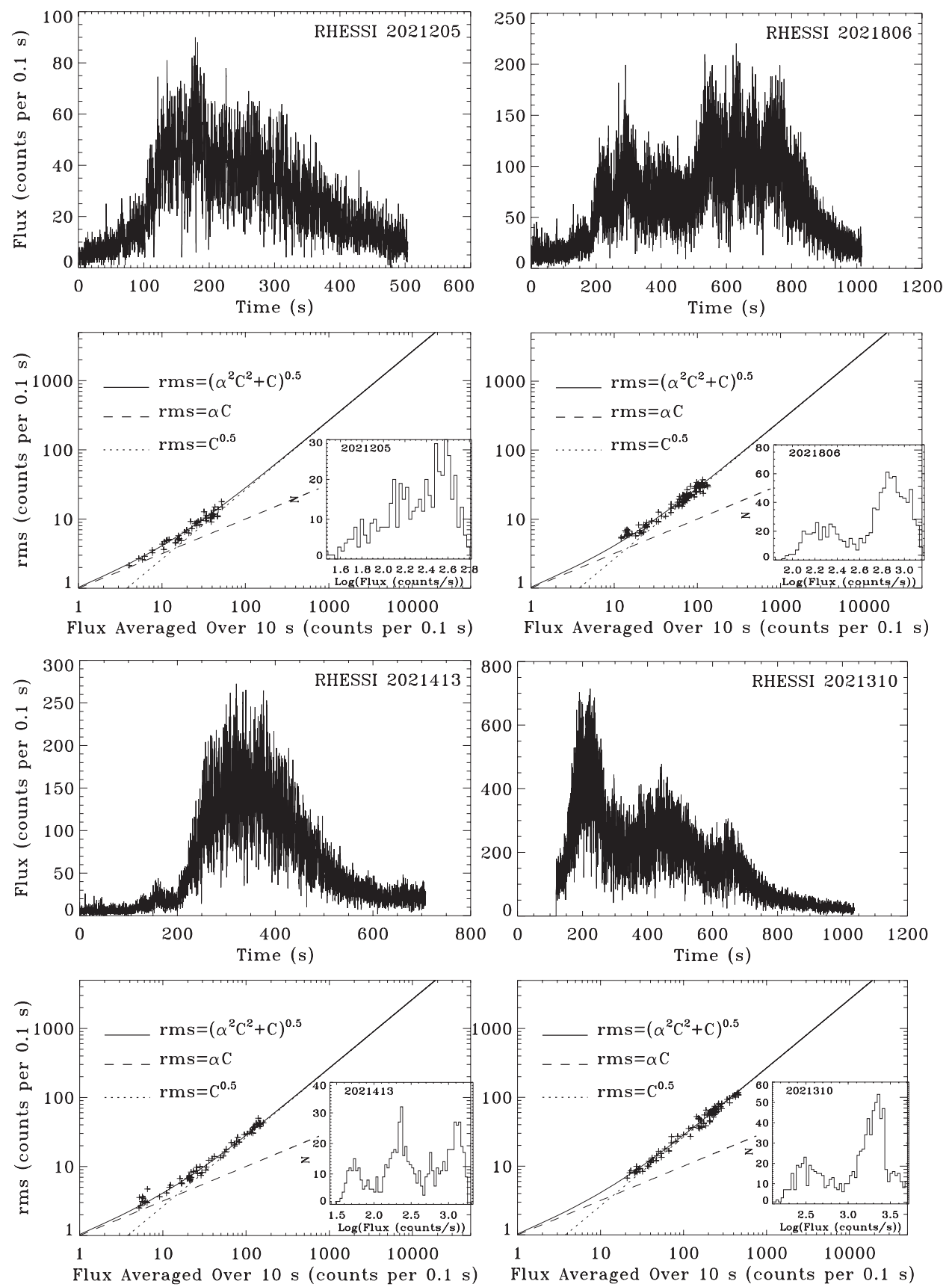

Figure 4. The lightcurves, flux-rms correlation and distribution of the logarithm of the flux for four individual solar X-ray flares. It should be noted $\alpha=0.27$ for all of them, and that their distributions of the logarithm of the flux are made of one or several normal distributions.

Cygnus X-1 with more data). The crosses are for the average values of rms for each flux interval divided uniformly in linear scale due to the narrow flux range. Compared to the flux-rms relation for RHESSI solar X-ray flares, the model describing the correlation is 

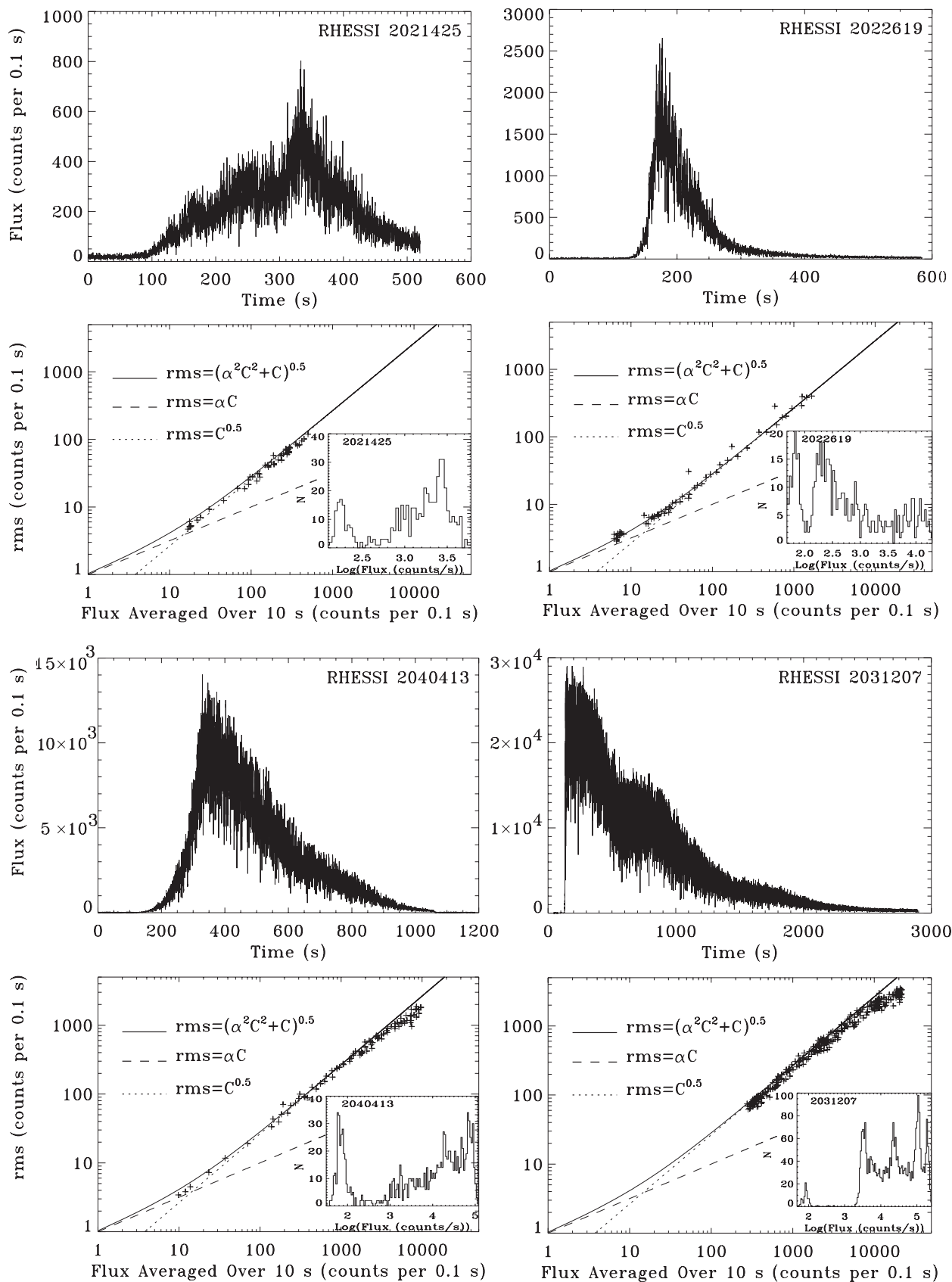

Figure 5. Continuation of Fig. 4.

only slightly more complicated,

$$
\mathrm{rms}^{2}=(\alpha F)^{2}+C, F=C-C_{0},
$$

where the total count $C$ consists of a constant term $C_{0}=57$ and a variable flux with $\mathrm{rms}=0.38 \times F$ over the Poisson counting fluctuations. Note that $C_{0}=57$ is well above the PCA background level, and is thus an intrinsic component from Cygnus X-1. The inset in Fig. 9 shows the log-normal flux distribution of Cygnus X-1. It has been known that 

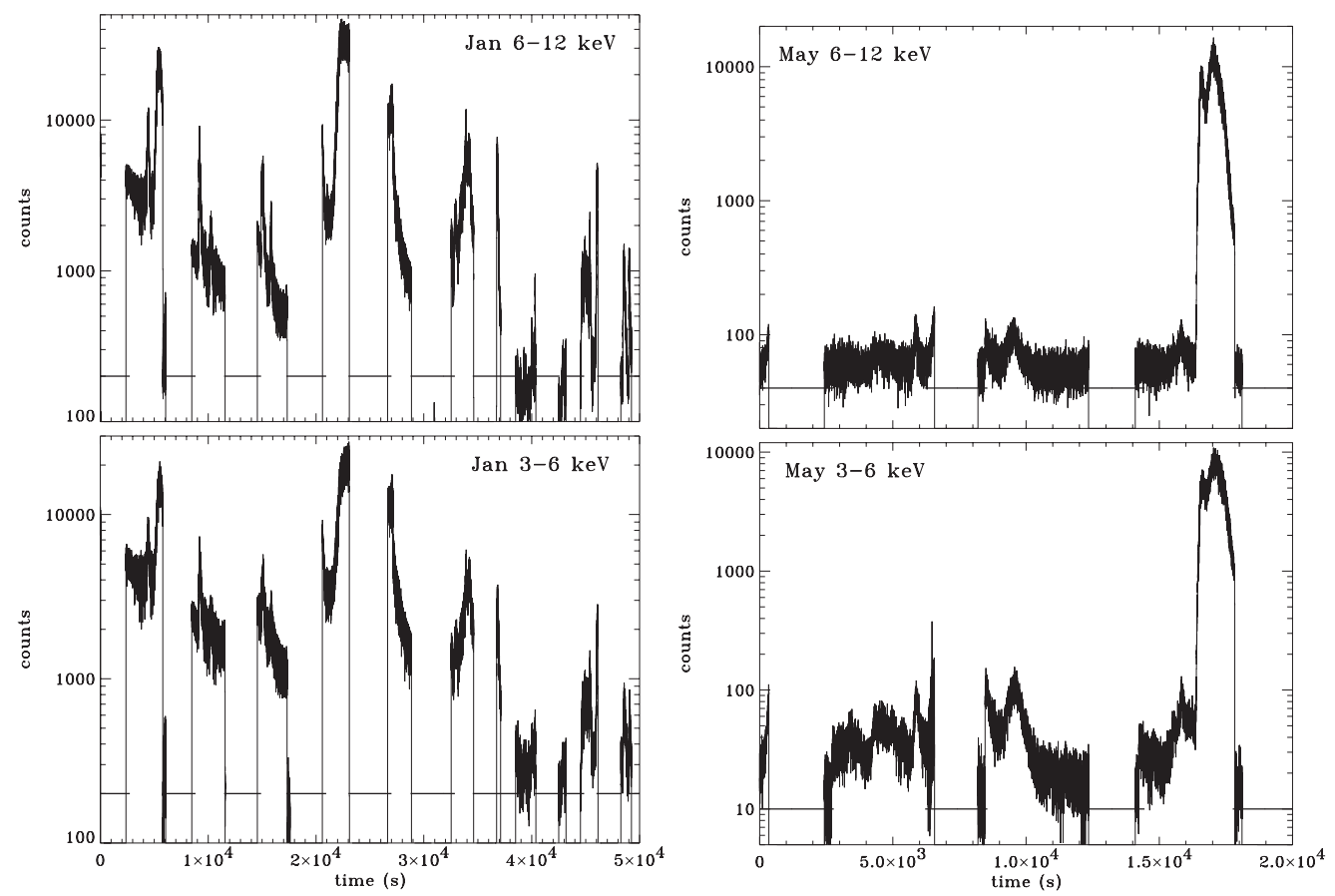

Figure 6. Solar X-ray lightcurves in two energy bands with $1 \mathrm{~s}$ resolution, on 2004-Jan-1.0 (left panels) for 50,000 consecutive seconds and on 2004-May-1.0 (right panels) for 20,000 consecutive seconds. Horizontal straight bars near the bottom in each panel mark data gaps, i.e., 'night' times for the RHESSI satellite.
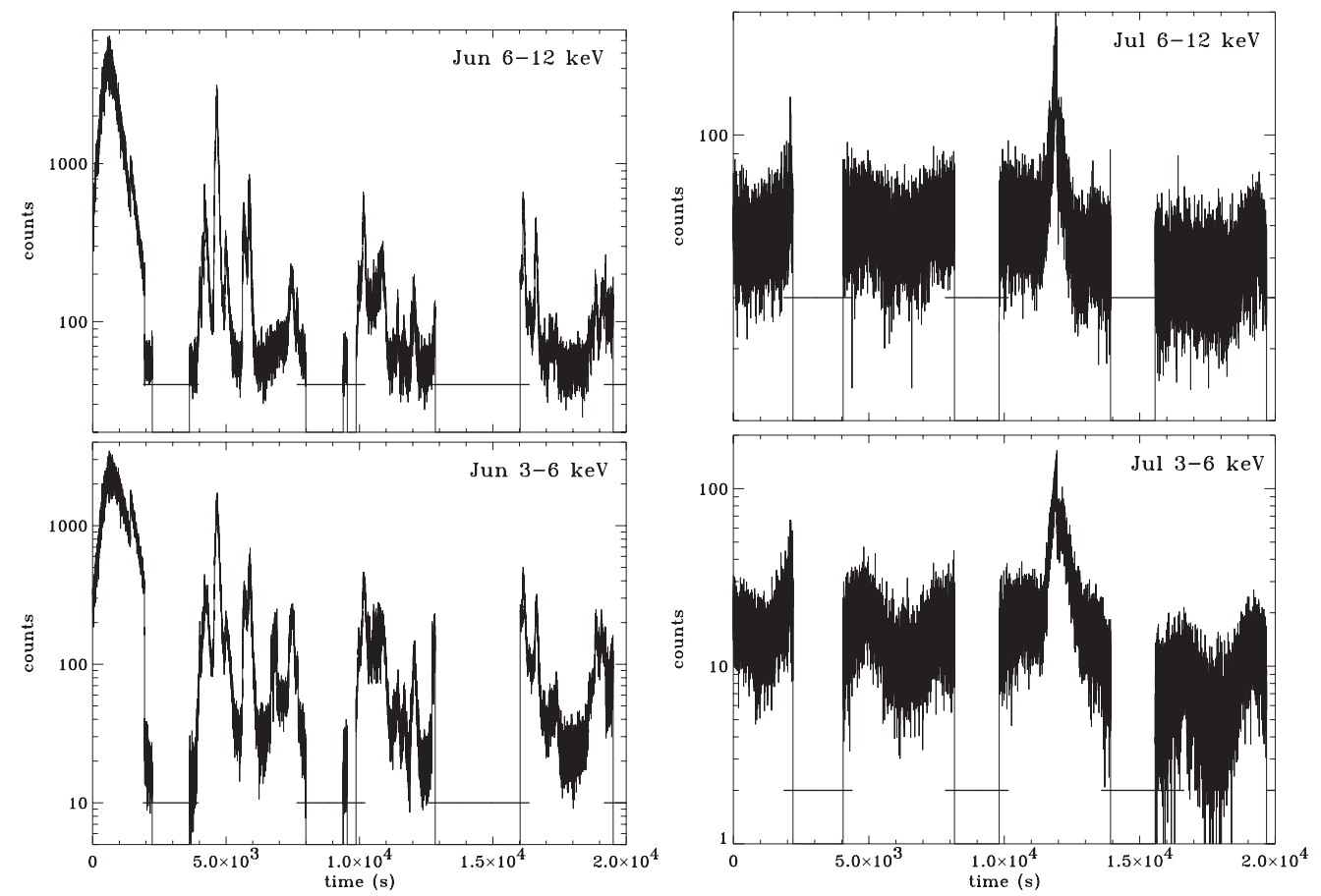

Figure 7. Solar X-ray lightcurves in two energy bands with $1 \mathrm{~s}$ resolution, on 2004-Jun-1.0(left panels) for 20,000 consecutive seconds and on 2004-Jul-1.0 (right panels) for 20,000 consecutive seconds. Horizontal straight bars near the bottom in each panel mark data gaps, i.e., 'night' times for the RHESSI satellite. 


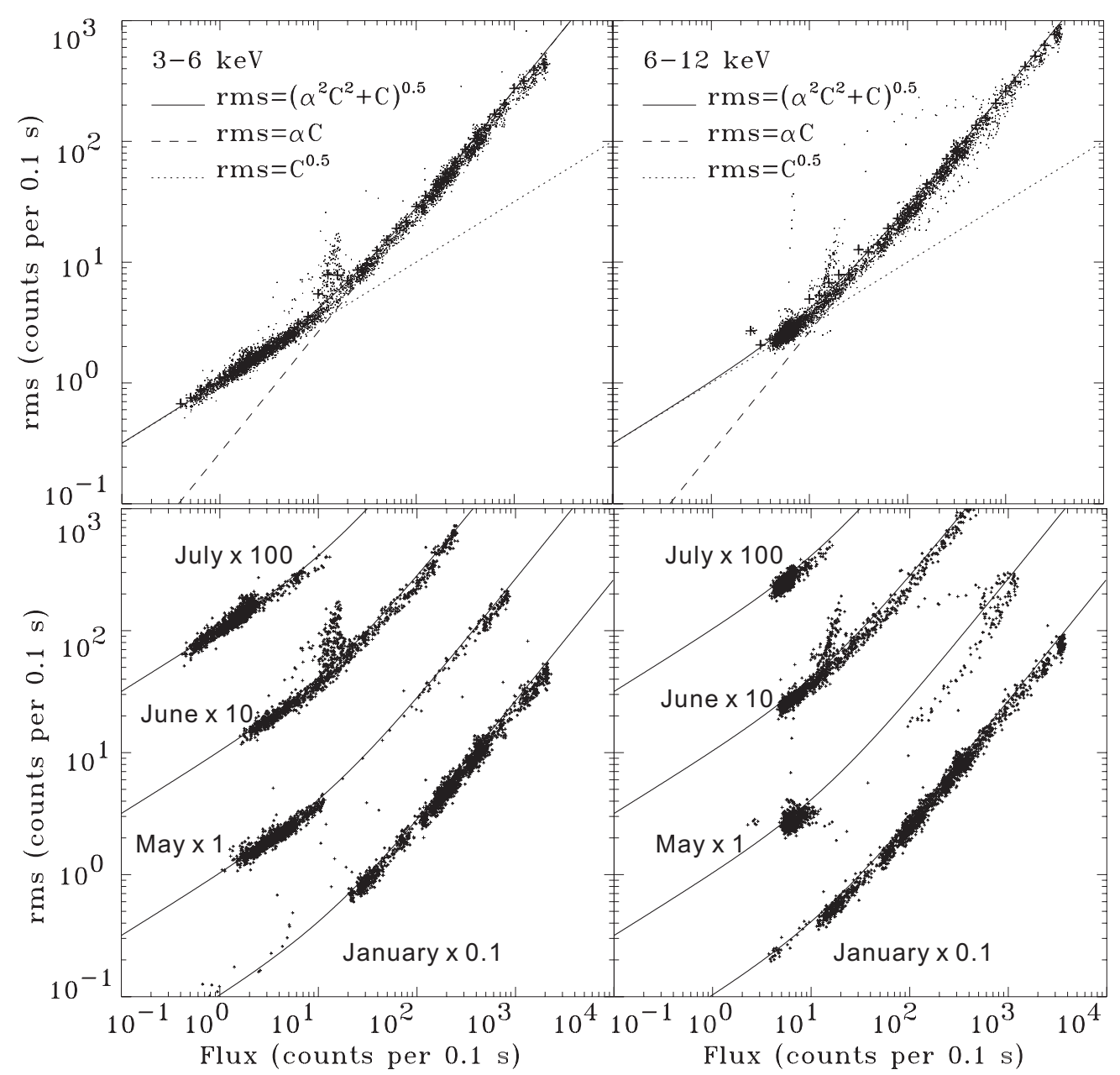

Figure 8. Upper panels: The relationship between flux and rms for all four segments of lightcurves in two energy bands. Each point in the scatter plots is for a $10 \mathrm{~s}$ interval of data with $0.1 \mathrm{~s}$ resolution. Lower panels: The flux-rms relations for the four lightcurves are shown separately; the data and model lines for January, June and July are shifted vertically for clarity. Clearly all four lightcurves have consistent flux-rms relations.

similar linear flux-rms relation exists for neutron star (Uttley 2004) and black hole binaries (Uttley \& McHardy 2001), as well as for supermassive black hole binaries (Edelson et al. 2002, Vaughan et al. 2003a, Vaughan et al. 2003b).

In Figs. 10 \& 11 the lightcurve and flux-rms relation for GRB 940217, detected with the BATSE instrument onboard the Compton Gamma-ray Observatory†, are shown for comparison. Equation (3.3) provides a consistent description for the flux-rms relation with $\alpha=0.25$ and $C_{0}=600$. Compared to the flux-rms relation for Cygnus X-1, $C_{0}=600$ is entirely due to the background level of the BATSE instrument. Therefore, the flux-rms relation for GRB 940217 is described by exactly the same model for RHESSI solar X-ray flares, if the different instrumental background levels are taken into account. 


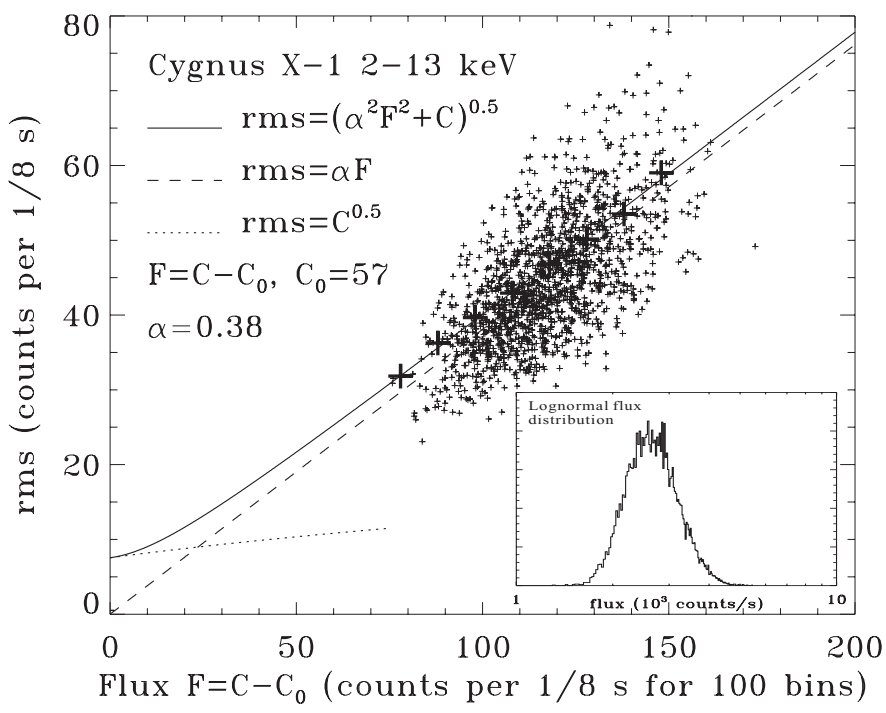

Figure 9. The flux-rms relation for the canonical stellar mass black hole binary Cygnus X-1 in the $2-13 \mathrm{keV}$, with data collected with the PCA instrument onboard the Rossi X-ray Timing Explorer from 1996-10-23-18:30:24 to 1996-10-24-02:30:26. The crosses for the average values of rms for each flux interval divided uniformly in linear scale due to the narrow flux range. Note counts are for per $1 / 8 \mathrm{~s}$ time bin; each point in the scatter plots is for a $10 \mathrm{~s}$ interval of data with $1 / 8 \mathrm{~s}$ resolution. The inset shows its $\log$-normal flux distribution.

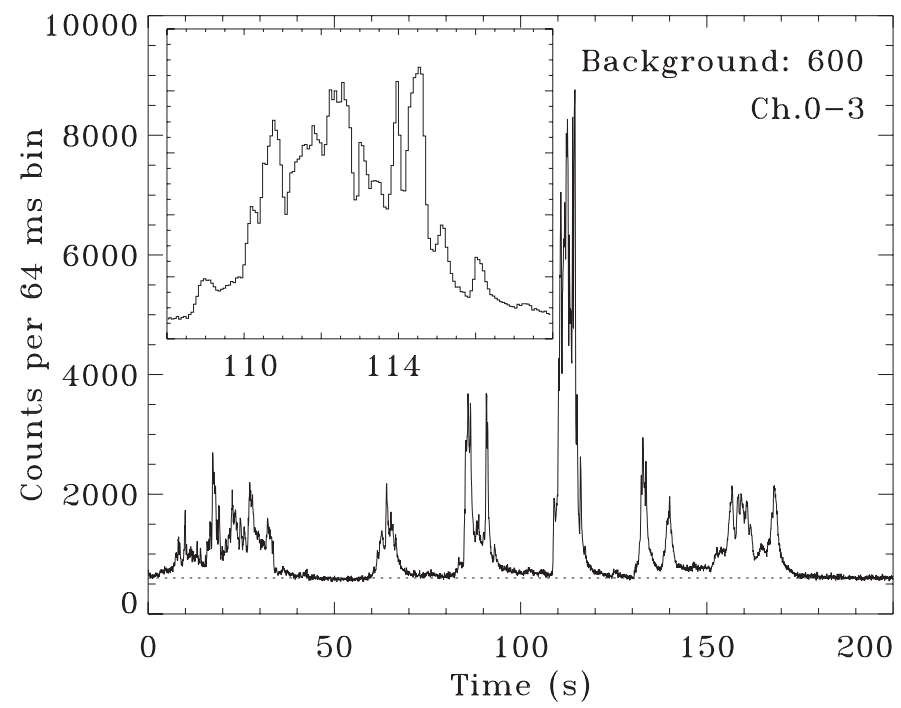

Figure 10. The lightcurve of GRB 940217 (trigger No. 2831) in channels 0-3 for the 16 channel data, i.e., between 13 to $54 \mathrm{keV}$, collected with the BATSE instrument onboard the Compton Gamma-ray Observatory. The inset shows clear variability above Poisson fluctuations. Note the background level is about 600 per $64 \mathrm{~ms}$ bin.

We chose to use GRB 940217 as an example of $\gamma$-ray bursts, because of its large dynamical range of flux and very long duration (thus many photons are available for a statistically meaningful correlation for a single $\gamma$-ray burst), as well as its very complex light curve morphology, for illustrating the robustness of the correlation. We comment in passing that GRB 940217 is a very important gamma-ray burst in its own right, 


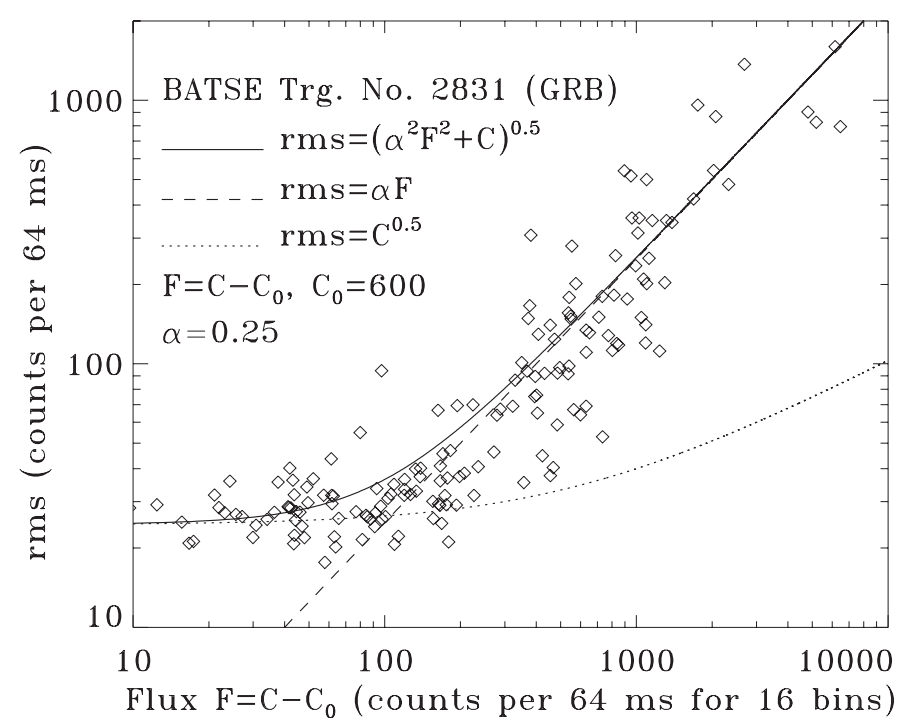

Figure 11. The flux-rms relation for the lightcurve of GRB 940217. The model is the same as equation (3.3).

because of its long-lasting high energy afterglow emission detected; > $30 \mathrm{MeV}$ photons were recorded for about $5400 \mathrm{~s}$, including an $18 \mathrm{GeV}$ photon about $4500 \mathrm{~s}$ after the low energy $\gamma$-ray emission had ended in the BATSE band as shown in Fig. 10 (Hurley et al. 1994). However, despite of the similar rms-flux correlation between GRB 940217 and solar flares, it is not meaningful to include multiple $\gamma$-ray bursts in one correlation plot, because different gamma-ray bursts are expected to have quite different characteristic time scales, since it is now known that long duration gamma-ray bursts have a large range of relativistic beaming factors and are produced in the whole Universe up to at least the redshift of 10 (see, e.g., Lin, Zhang \& Li 2004, Zhang 2007).

\subsection{Further comments on the flux-rms correlations}

For the X-ray flux-rms correlations from the Sun, Cygnus X-1, and GRB 940217, about the only qualitative differences are: $(a)$ There is a non-negligible constant flux from the total X-ray flux of Cygnus X-1 but not from the Sun or GRB 940217; (b) The range of rms variations from the Sun and GRB 940217 covers over several orders of magnitudes, but only about a factor of two for Cygnus X-1; $(c)$ The flux distribution for each solar flare is made of one or more log-normal peaks, in comparison for only a single log-normal peak for that of Cygnus X-1. For the Sun and GRB 940217, even the portion of Poisson noise domination is seen clearly. It is commonly known that solar X-rays are produced from flare regions on solar surface, prompt $\mathrm{X} / \gamma$-rays from $\gamma$-ray bursts (at least for long duration gamma-ray bursts such as GRB 940217) originate from the colliding relativistic jets (the so-called internal shock model, see, e.g., Piran 2004 for a recent review and references therein), and X-rays from black hole binaries are emitted from the accretion disks around their central black holes. The remarkable common X-ray flux variations between the apparently very different systems at different scales suggest that there should be a common and dominating mechanism operating in all of them, although we cannot exclude the possibility that different physical mechanisms may generate very similar X-ray flux variations. Because the Sun is our closest astrophysical laboratory from which we can obtain a wealth of information, in particular the direct measurements of its magnetic 
field topology and activities, understanding the solar X-ray production mechanism may be a key to revealing the black hole accretion disks and gamma-ray burst jets.

The success of this simple model with only one parameter in describing the very complex solar X-ray flares and continuous lightcurves, observed at well separated times in two energy bands, implies that the linear flux-rms relationship is very helpful for our understanding of X-ray emission from the Sun, and perhaps also the corona heating problem. Previously EUV and X-ray variability in the pixels of focal plane detectors of TRACE and YOHKOH have been analysed in order to probe the contribution of the speculated 'nano'-flares to the solar corona heating (Shimizu \& Tsuneta 1997, Krucker \& Benz 1998, Aschwanden et al. 2000a, Aschwanden et al. 2000b, Parnell \& Jupp 2000, Katsukawa \& Tsuneta 2001, Benz \& Krucker 2002, Katsukawa 2003). However it is still not settled if the amount of 'nano'-flares can fulfill the requirement of Parker's conjecture (Walsh 2003). Nevertheless, these studies have indicated that probing X-ray variability is probably so far the best way for studying this outstanding problem in astrophysics. However due to the very limited counting statistics in those previous studies, no individual counting excess above noise fluctuation can be identified as real flaring events. It is thus uncertain if these variability studies have revealed unambiguously 'nano-'flares as extrapolated smaller events from observed solar flares.

It is interesting to note that the detected X-ray variability, with the Yohkoh Soft X-ray Telescope, is related to its mean flux as (Katsukawa \& Tsuneta 2001), $\operatorname{rms} \propto F^{0.93 \pm 0.10}$, in good agreement with the linear relationship shown here with RHESSI data for Xray variability including all flaring events detected in the X-ray lightcurves. This should be compared to the case of Cygnus X-1 in which the X-ray variability is also mostly composed of unidentifiable flaring events, despite that occasionally very strong flares are detectable (Gierlinski\& Zdziarski 2003), which can be considered as analogy of solar flares in active regions of the Sun. Therefore, the results shown in Fig. 8 for the flux-rms linear relationship over several orders of magnitudes of X-ray flux variations from the Sun can be regarded as a missing link between the extremely violent flaring events from the Sun, and X-ray flux variations with small relative amplitudes from the Sun and from accreting neutron star and black hole binaries systems.

These results therefore demonstrate that common dynamical physical processes may dominate the X-ray emission from the Sun, $\gamma$-ray bursts, accreting neutron star and black hole binaries, as well as in accreting supermassive black hole systems (Uttley et al. 2005). Since accretion flow does not exist in the Sun, this may rule out immediately the fluctuating accretion flow model (Lyubarskii 1997, Kotov 2001) for this type of dynamical properties occurring in neutron star and black hole binaries with accretion disks. Because essentially all other models for X-ray emission from neutron star and black hole systems have already been ruled out by such dynamical behaviours (Uttley et al. 2005), new physical models for them may be required, which should have common mechanisms to X-ray emission from the Sun. We, therefore, probably should return to the solar flare-like models for accreting neutron star and black hole systems, as well as to $\gamma$-ray bursts.

\section{Common physical mechanisms: self-organized criticality, percolation and dynamical driving}

Then what are the possible common physical mechanisms? Magnetic energy releases, perhaps due to magnetic reconnections, should be the engines in all these systems (Nayakshin \& Melia 1997, di Matteo et al. 1999, Poutanen \& Fabian 1999, Zhang et al. 2000, Liu et al. 2003, Wang et al. 2004, Liu \& Li 2004, Priest \& Forbes 2002). For the Sun, an emerging flux and reconnection model has been proposed for the triggering of the 
coronal mass ejections (Chen \& Shibata 2000). In addition, some small scale activities, such as Ellerman bombs, and type II white-light flares are possibly the results of magnetic reconnection in the solar lower atmosphere (Chen, Fang \& Ding 2001). The avalanche mechanisms (Charbonneau et al. 2001) driven by self-organized criticality (Bak et al. 1988, Christensen et al. 1992) have been widely invoked to explain previously observed distributions of solar flare peak flux and event interval, as well as timing properties in accreting black hole systems (Mineshige et al. 1994, Takeuchi et al. 1995). The multiplicative nature of the generated events in the self-organized criticality model can produce the observed flux-rms linear relationship. However the simple self-organized criticality model also predicts power-law distributions of flux, which is not found in accreting neutron star and black hole systems, and in fact also not verified satisfactorily to the whole range of amplitudes of solar flares; the power-law flux distribution of solar flares is the basic idea of Parker's conjecture (Parker 1983, Parker 1988, Parker 1991). As shown in Figs. 4 \& 5 , the flux distributions of individual solar flares consist of multiple log-normal peaks, rather than a single power-law.

This mismatch between observed and predicted flux distributions may be due to oversimplified self-organized criticality models which only describe events occurring locally. However X-ray photons from accreting neutron star and black hole systems cannot come from only a localized self-organized criticality spot, yet events occurred are still interrelated as indicated by the multiplicative property. Therefore multiple spots or a major part of the whole inner accretion disk region responsible for X-ray emission must be inter-organized together somehow; this is further supported by the fact that only about $30 \%$ or less of its total X-ray flux does not follow the flux-rms relationship in Cygnus X-1 (see Fig. 9), i.e., only this $30 \%$ flux is the superposition of many randomly occurring and independent emission events. The RHESSI solar X-ray lightcurves we analysed here are collected from the whole Sun exposed to us, and its non-varying X-ray flux is very low, suggesting that essentially all detectable X-ray emission in a time series over the whole Sun is produced from many inter-related X-ray emission regions. In fact, it has already been observed that many active regions well separated and spread nearly over the whole solar surface show networked activities; these regions are now known to be connected by large scale magnetic field lines (Zhang et al. 2006), as shown in Fig. 12.

In a simulation study of particle acceleration during solar flares, Vlahos et al. 2004 have found that multi-scale magnetic fields are involved, which may be described mathematically by the percolation model of Albert \& Barabasi 2002, as illustrated in Fig. 13. In the percolation model, two nodes are connected by an edge with a certain probability. If the probability is below the percolation threshold, the connected nodes form small and local clusters. In this case, most nodes will act independently, and thus their total contributions will be additive, rather than multiplicative. On the other hand, if the connection threshold is above the percolation threshold, global and large clusters are formed, and thus most nodes will act inter-connectively. In this case their total contributions will be multiplicative, because the action of each node will influence many other nodes, even if they are well separated spatially. Such networked activities or fluctuations can be studied by identifying the dominating driving mechanisms, i.e., internal or external driving. If the connection probability between two neighboring nodes is below the percolation threshold, the dynamical system will be dominated by internal driving, or otherwise dominated by external driving.

Following Argollo de Menezes \& Barabasi (2004), for a dynamical system, we can record the time dependent activity of $N$ components, expressed by a time series $\left\{f_{i}(t)\right\}$, $t=1, \ldots, T$ and $i=1, \ldots, N$. As each time series reflects the joint contribution from the system's internal dynamics and external fluctuations, we assume that we can separate 


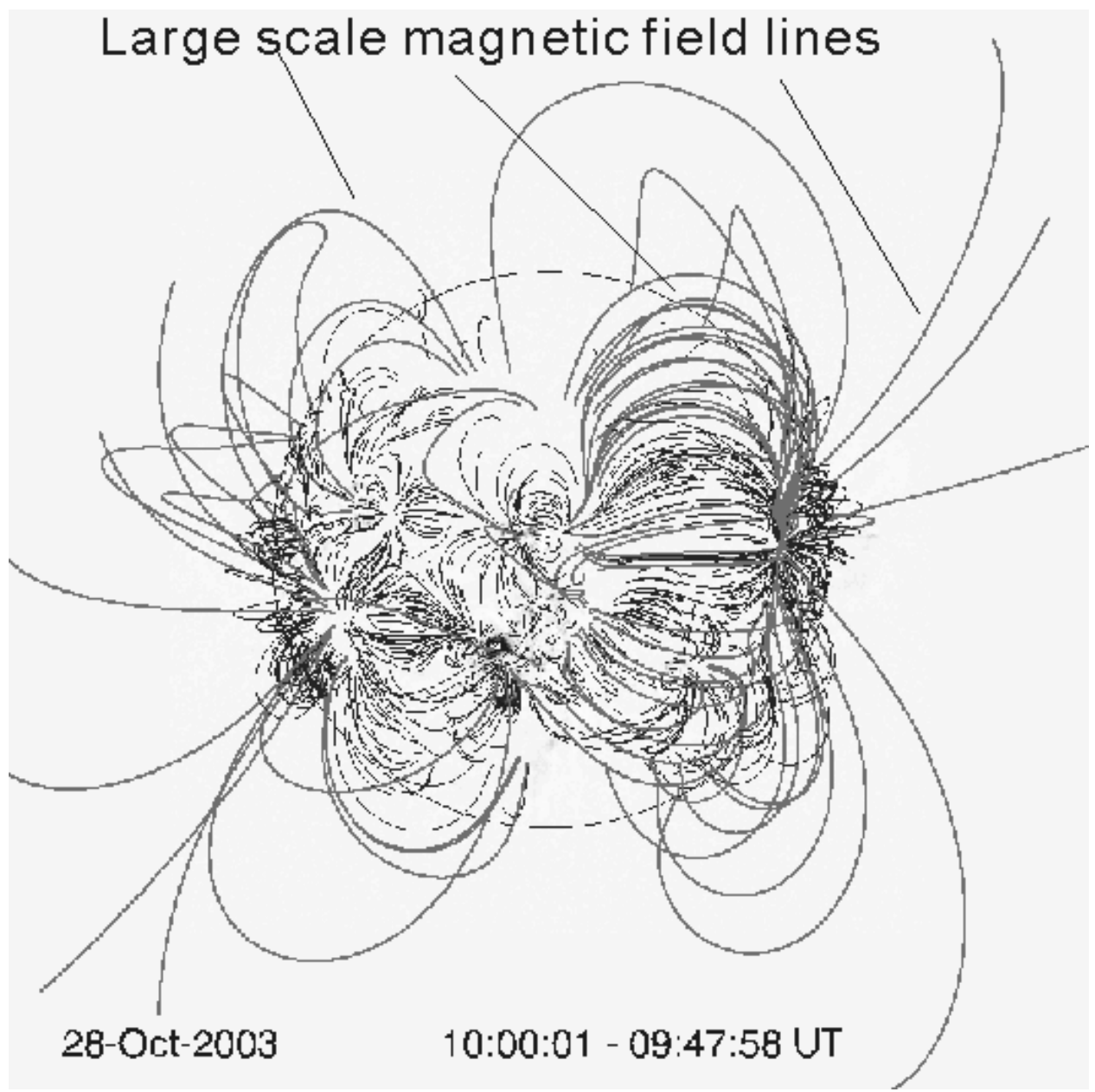

Figure 12. Solar magnetic field topology during the major solar activities on October 28th, 2003. Many active regions are connected by complex magnetic field lines. (This figure is adapted from Zhang et al. 2006.)

the two contributions by writing

$$
f_{i}(t)=f_{i}^{\text {int }}(t)+f_{i}^{e x t}(t) .
$$

It has been shown by Argollo de Menezes \& Barabasi (2004) that the fluctuation or rms is linearly proportional to $f$ if the dynamical system is externally driving, or the rms is proportional to $\sqrt{f}$ if the dynamical system is internally driving.

Since the dominating component of the X-ray flux fluctuation or rms is proportional to flux, for solar X-ray flares and continuous light curves, the $\mathrm{X} / \gamma$-ray lightcurve of GRB 940217, accreting neutron star and black hole systems, we can infer that their Xray production mechanisms are all dominated by external driving dynamics. We thus suggest that perhaps similar large scale magnetic field topology also exists in the accretion disks around neutron stars and black holes, and also maybe in $\gamma$-ray bursters. We comment in passing that no current $\gamma$-ray burst model involves inter-connected emission nodes. However, contrary to the widely accepted internal shock model of prompt $\gamma$-ray emissions for $\gamma$-ray bursts, an alternative model, based on electrodynamic accretion mod- 


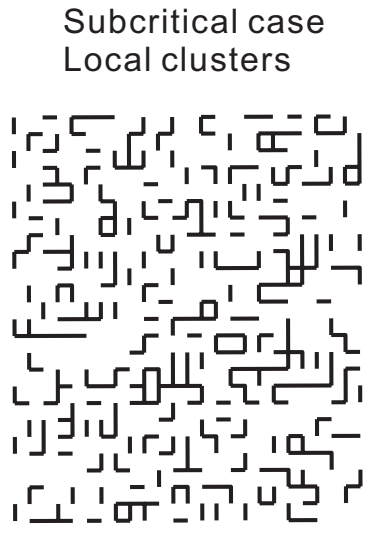

$p=0.315$
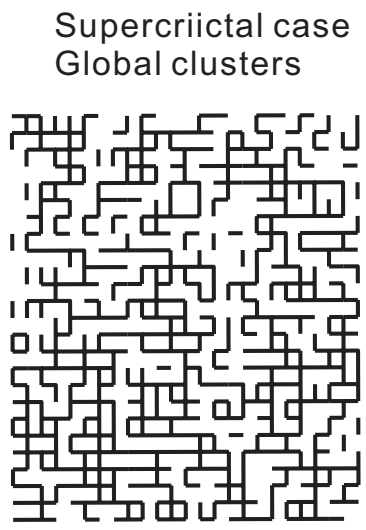

$p=0.525$

Figure 13. Illustration of the percolation model of Albert \& Barabasi 2002. Two nodes are connected by an edge with probability $p$. For $p=0.315$ (left), which is below the percolation threshold $p_{c}=0.5$, the connected nodes form small and local clusters. For $p=0.525$ (right), which is above the percolation threshold, the global and large cluster are formed. (This figure is adapted from Albert \& Barabasi 2002.)

els in which the electromagnetic process turns rotational energy into particle energy in a pulsarlike mechanism (Katz 1997), has been used to unite jets in $\gamma$-ray bursts, accreting supermassive and stellar mass black hole systems (Katz 2006). It has also been suggested a magnetic field dominated outflow model for GRBs is preferred, based on data from the dedicated $\gamma$-ray burst satellite Swift (Kumar et al. 2007). These results are in qualitative agreement with our common energy release picture for these different types of objects at very different scales, suggesting that it may be necessary to re-examine seriously the currently accepted standard $\gamma$-ray burst internal shock models, by investigating energy release processes dominated and connected by complex magnetic field topology.

Although a component of rms proportional to the square-root of total counts is identified in all of them, especially when the total counts are small, this component is not due to internal driving, because this component can be completely described as Poisson counting fluctuations. This means that currently no internal driving dynamics has been detected in any of them. It is interesting to note that the individual solar flares show the same flux-rms linearity as for the total continuous lightcurves of all those systems, indicating that each individual solar X-ray flare is produced from many different interconnected emission nodes or elements, if the percolation model is also applicable here. It is thus reasonable to assume that regardless what triggers the initial onset of a solar $\mathrm{X}$-ray flare, the event immediately spreads out to many nodes or elements. Imaging observations of X-ray flares have identified magnetic loops and their foot-points as X-ray and hard X-ray production sites. Therefore it will be important to find the relationship between the above inferred inter-connected X-ray emission nodes or elements and observed magnetic loops and their foot-points.

\section{Concluding remarks}

In section 1, I briefly categorized different research methods in astronomy and emphasized the importance of using the Sun as an astrophysical laboratory to study many astrophysical phenomena across vastly different scales, but appearing to be similar to 
what occurring in the Sun. I call this research discipline "Applied Solar Astrophysics". A beautiful example of the progress in this discipline is the use of magnetic reconnection model by Dai et al. 2006 to explain the late X-ray flux re-brightening in the X-ray afterglow of a short $\gamma$-ray burst, which is believed to have produced a spinning young neutron star after two neutron stars merged together (this merging event triggered the initial onset of the short $\gamma$-ray burst).

In section 2, I briefly summarized several phenomena: (1) the atmospheric structures around accreting black holes which are similar to solar atmosphere; (2) astrophysical jets from many different kinds of astrophysical systems, whose production mechanisms involve differential rotation and twisted magnetic fields similar to magnetic reconnections in the Sun for solar flares and coronal mass ejection; (3) similar triple-ring structure in SN 1987A and Virgo cluster which are understood to be related to rotating large scale magnetic field topology and winds, again similar to the solar magnetic fields and solar winds. Clearly our understanding of related solar phenomena are important for studying these similar phenomena at different scales.

In section 3, I focused on the similar non-linear dynamical properties of X-ray variations of solar flares, a stellar mass black hole binary Cygnus X-1 and a $\gamma$-ray burst GRB 940217. The remarkable consistency of the underlying non-linear dynamics model for these three kinds of apparently very different astrophysical systems at different scales suggests that many X-ray emission nodes or elements are somehow inter-connected and act in response to common triggering. Again using the Sun as an astrophysical laboratory, we suggest that complicated magnetic field topology across many different scales in each system plays an important role in connecting many X-ray emission nodes or elements together. It is possible that these individual nodes produce 'nano'-flares required for heating the solar corona.

However previous and current solar X-ray instruments do not have the required capability to resolve individual X-ray flares or the quiet Sun X-ray lightcurves into many 'nano'-flares. We suggest that a future Solar X-ray Timing and Imaging) (SXTI) instrument with a large effective area and direct high-resolution imaging capability is needed to further resolve the fine timing structures of X-ray flares, i.e., inter-connected individual 'nano'-flares, to study the development of solar flares in fine details, and overcome the Poisson counting fluctuations with a high counting rate during the quiet Sun, in order to identify the internal driving component, which may be made of isolated 'nano'-flares.

Although it is beyond the scope of this report for proposing a conceptual design for the SXTI telescope, here we outline only the basic requirements: (1) an effective area of $>100 \mathrm{~cm}^{2}$ at a few $\mathrm{keV}$ to be compared with the $30 \mathrm{~cm}^{2}$ effective area at $10 \mathrm{keV}$ for RHESSI, however RHESSI relies on rotation modulation imaging and thus cannot offer simultaneous imaging and high timing resolution capability; (2) direct imaging angular resolution of $<1$ arcsec, to be compared with HINODE (Solar-B)'s X-ray telescope† which has 2 arcsec direct imaging resolution, but with only $1 \mathrm{~cm}^{2}$ effective area at $0.523 \mathrm{keV}$ and $2 \mathrm{~s}$ timing resolution; and (3) timing resolution $<10 \mathrm{~ms}$ which requires advanced X-ray CCDs, such as that in current developments (e.g., Zhang et al. 2007).

For comparison the currently operating Chandra X-ray telescopeł has a peak effective area of about $800 \mathrm{~cm}^{2}$ at around $2 \mathrm{keV}$, with a direct imaging resolution of 0.5 arcsec and each X-ray CCD's full-frame readout time of around $3.3 \mathrm{~s}$. Although telescopes pointed at the Sun involve significantly more complex technical difficulties, it is possible that a solar X-ray observatory with the above proposed $S X T I$ will be technically feasible within

$\dagger$ http://solar-b.nao.ac.jp/xrt_e/fact_sheet_e.shtml $\ddagger$ http://chandra.harvard.edu/about/science_instruments.html 
the near future. With such an instrument, our understanding of solar X-ray production mechanism, and subsequently many currently open issues on solar magnetic fields, particle acceleration and coronal heating, etc., will be advanced significantly. Because of many astrophysical phenomena at different scales should have similar underlying physical processes to what occurring in the Sun, we should also make significant progresses towards understanding many important astrophysical problems. Therefore "Applied Solar Astrophysics" has a bright future.

\section{Acknowledgements}

I shall thank Cheng Fang of Nanjing University for nominating me, the IAU Executive Committee for inviting me, as one of the four discourse speakers at the XXVI ${ }^{t h}$ IAU GA in Prague, Czech Republic, August 2006, and Ding-Qiang Su of Nanjing University, who was then the President of the Chinese Astronomical Society, for encouragements and advises during my preparation of this invited discourse. I also appreciate discussions with Bob Lin of UC Berkeley and Don Melrose of University of Sydney during the 26th IAU GA on RHESSI data analysis and solar particle accelerations, respectively. The many kind compliments and stimulating discussions from the audience in the discourse are the best rewards for my discourse, which has indeed taken me a lot of time and efforts to prepare for.

I also thank Shui Wang of University of Science and Technology of China for discussions on magnetic reconnections, Jun-Han You of Shanghai Jiao-Tong University for discussions on radiation mechanisms, Wei-Qun Gan of Purple Mountain Observatory for advises on RHESSI data analysis and conversations on solar flares, Jing-Xiu Wang of National Astronomical Observatories of China and Louise Harra of Mullard Space Science Laboratory of University College London for exchanges on solar physics, Jian-Min Wang of Institute of High Energy Physics for many discussions on accretion physics, and many of my previous collaborators and formal students for the privilege and fun of working with them and for their important contributions to our works discussed and/or referenced in this report. My student Shi-Cao Tang of Tsinghua University is greatly acknowledged for assisting me in most of the data analysis work shown in Figs. 3-11 of this report. Finally Cheng Fang, Zi-Gao Dai \& Peng-Fei Chen of Nanjing University, Tan Lu of Purple Mountain Observatory, Jun Lin of Yunnan Observatory, Jing-Xiu Wang of National Astronomical Observatories of China, Phil Uttley of Southampton University and Simon Vaughan of Leicester University are thanked for comments and suggestions on the manuscript of this report.

This work is supported in part by the Ministry of Education of China, Directional Research Project of the Chinese Academy of Sciences and by the National Natural Science Foundation of China under project no. 10521001, 10327301, 10233010 and 10233030.

\section{References}

Albert, R., \& Barabasi, A.L. 2002, Rev. Mod. Phys., 74, 47

Argollo de Menezes, M., \& Barabasi, A.L. 2004, Phys. Rev. Lett., 93, 068701

Aschenbach, B., Grosso, N., Porquet, D., \& Predehl, P. 2004, A\&A, 417,71

Aschwanden, M.J., Nightingale, R.W., Tarbell, T.D., \& Wolfson, C.J. 2000a, ApJ, 535, 1027

Aschwanden, M.J., Tarbell, T.D., Nightingale, R.W., et al. 2000b, ApJ, 535, 1047

Bak, P., Tang, C., \& Wiesenfeld, K. 1988, Phys. Rev. A, 38, 364

Bassani, L., Malaguti, G., Paciesas, W.S., Palumbo, G.G.C., \& Zhang, S.N. 1996, A $\& A S$, 120C, 559

Burrows, C.J. 1995, ApJ, 452, 680

Benz, A.O., \& Krucker, S. 2002, ApJ, 568, 413 
Charbonneau, P., McIntosh, S.W., Liu, H.-L., \& Bogdan, T.J. 2001, Sol. Phys., 203, 321

Chen, P.F., \& Shibata, K. 2000, ApJ, 545, 524

Chen, P.F., Fang, C., \& Ding, M.D. 2001, ChJAA, 1, 176

Chen, X., Zhang, S.N., \& Ding, G.Q. 2006, ApJ, 650, 299

Christensen, K., Olami, Z., \& Bak, P. 1992, Phys. Rev. Lett., 68, 2417

Cranmer, S.R., \& van Ballegooijen, A.A. 2005, ApJS, 156, 265

Dai, Z.G., Wang, X.Y., Wu, X.F., \& Zhang, B. 2006, Science, 311, 1127

Dennis, B.R., Hudson, H.S., \& Krucker, S. 2005, in: Proc. CESRA Workshop 2004, Isle of Skye, Scotland Review of selected RHESSI solar results

di Matteo, T., Celotti, A., \& Fabian, A.C. 1999, MNRAS, 304, 809

Doi, K. 1978, Nature, 275, 197

Edelson, R., Turner, T.J., Pounds, K.A., et al. 2002, ApJ, 568, 610

Feng, H., Zhang, S., Lou, Y., \& Li, T. 2004, ApJ (Letters), 607, L95

Gierlinski, M., \& Zdziarski, A.A. 2003, MNRAS (Letters) 343, L84

Haardt, F., Maraschi, L., \& Ghisellini, G. 1997, ApJ, 476, 620

Harmon, B.A, Wilson, C.A., Zhang, S.N., Paciesas, W.S., Fishman, G.J., Hjellming, R.M., Rupen, M.P., Scott, D.M., Briggs, M.S., \& Rubin, B.C. 1995, Nature, 374, 703

Hawley, J.F., Balbus, S.A., \& Winters, W.F. 1999, ApJ, 518, 394

Hurley, K., Dingus, B.L., Mukherjee, R., et al. 1994, Nature, 372, 652

Katsukawa, Y. 2003, PASJ, 55, 1025

Katsukawa, Y., \& Tsuneta, S. 2001, ApJ, 557, 343

Katz, J.I. 1997, ApJ, 490, 633

Katz, J.I. 2006, [astro-ph/0603772]

Klimchuk, J.A. 2006, Sol. Phys., 234, 41

Kotov, O., Churazov, E., \& Gilfanov, M. 2001, MNRAS, 327, 799

Krucker, S., \& Benz, A.O. 1998, ApJ, 501, L213

Kubota, A., Makishima, K., \& Ebisawa, K. 2001, ApJ (Letters), 560, L147

Kumar, P., McMahon, E., Panaitescu, A., et al. 2007, MNRAS (Letters) 376, L57

Liang, E.P.T., \& Price, R.H. 1977, ApJ, 218, 247

Lin, R.P., Dennis, B.R., Hurford, G.J., et al. 2002, Sol. Phys., 210, 3

Lin, J.R., Zhang, S.N., \& Li, T.P. 2004, ApJ, 605, 819

Liu, C.Z., \& Li, T.P. 2004, ApJ, 611, 1084

Liu, B.F., Mineshige, S., \& Ohsuga, K. 2003, ApJ, 587, 571

Liu, Y., Zhang, S.N., \& Zhang, X.L. 2007, PASJ, 59, 185

Lyubarskii, Y.E. 1997, MNRAS, 292, 679

Margon, B., Bowyer, S., \& Stone, R.P.S. 1973, ApJ, 185, L113

Meier, D.L., Koide, K., \& Uchida, Y. 2001, Science, 291, 84

Markovskii, S.A., \& Hollweg, J.V. 2004, ApJ, 609, 1112

Mineshige, S., Ouchi, N.B., \& Nishimori, H. 1994, PASJ, 46, 97

Meszaros, P. 2001, Science, 291, 5

Mirabel, I.F., \& Rodriguez, L.F. 1998, Nature, 392, 673

Mirabel, I.F., \& Rodriguez, L.F. 2002, Sky \& Telescope, May 2002, 32

Miyamoto, S., Kitamoto, S., Mitsuda, K., \& Dotani, T. 1988, Nature, 336, 450

Nayakshin, S., \& Melia, F. 1997, ApJ (Letters), 490, L13

Negoro, H., Kitamoto, S., \& Mineshige, S. 2001, ApJ, 554, 528

Parker, E.N. 1983, ApJ, 264, 642

Parker, E.N. 1988, ApJ, 330, 474

Parker, E.N. 1991, ApJ, 376, 355

Parnell, C.E., \& Jupp, P.E. 2000, ApJ, 529, 554

Pevtsov, A.A. 2002, Sol. Phys., 207, 111

Piran, T. 2004, Rev. Mod. Phys., 76, 1143

Poutanen, J., \& Fabian, A.C. 1999, MNRAS (Letters), 306, L31

Priest, E.R., \& Forbes, T.G. 2002, A\&A Rev., 10, 313

Remillard, R.A., \& McClintock, J.E. 2006, ARA\& A, 44, 49 
Tang, S.C., \& Zhang, S.N. 2007, in preparation

Shakura, N.I., \& Sunyaev, R.A. 1973, A\&SA, 24, 337

Shimizu, T., \& Tsuneta, S. 1997, ApJ, 486, 1045

Takeuchi, M., Mineshige, S., \& Negoro, H. 1995, PASJ, 47, 617

Tanaka, T., \& Washimi, H. 2002, Science, 296, 321

Terrell, N.J.J. 1972, ApJ (Letters), 174, L35

Tingay, S.J., Jauncey, D.L., Preston, R.A., et al. 1995, Nature, 374, 141

Ueda, Y., Akiyama, M., Ohta, K., \& Miyaji, T. 2003, ApJ, 598, 886

Uttley, P. 2004, MNRAS (Letters), 347, L61

Uttley, P., \& McHardy, I.M. 2001, MNRAS (Letters), 323, L26

Uttley, P., McHardy, I.M., \& Vaughan, S. 2005, MNRAS, 359, 345

Vaughan, S., Fabian, A.C., \& Nandra, K. 2003a, MNRAS, 339, 1237

Vaughan, S., Edelson, R., Warwick, R.S., \& Uttley, P. 2003b, MNRAS, 345, 1271

Vlahos, L., Isliker, H., \& Lepreti, F. 2004, ApJ, 608, 504

Walsh, R.W., \& Ireland J. 2003, A $\&$ A Rev., 12, 1

Wang, J.-M., Watarai, K.-Y., \& Mineshige, S. 2004, ApJ (Letters), 607, L107

Wang, J., Zhang, J., Wang, T., Zhang, C., Liu, Y., Nitta, N., \& Slater, G.L. 2001, in: P. Brekke, B. Fleck \& J.B. Gurman (eds.), Recent Insights into the Physics of the Sun and Heliosphere: Highlights from SOHO and Other Space Missions, Proc. IAU Symp. No. 203 (San Francisco: ASP), p. 331

Wang, X.Y., Dai, Z.G., \& Lu, T. 2003, ApJ, 592, 347

Yamamoto, T.T., Kusano, K., Maeshiro, T., Yokoyama, T., \& Sakurai, T. 2005, ApJ, 624, 1072

Zhang, B. 2007, ChJAA, 7, 1

Zhang, C., Lechner, P., Lutz, G., Porro, M., Richter, R., Treis, J., Struder, L., \& Zhang, S.N. 2006, Nucl. Instr. \& Meth. A, 568, 207

Zhang, S.N., Wilson, C.A., Harmon, B.A., et al. 1994, IAUC 6046

Zhang, S.N., Harmon, B.A., Paciesas, W.S., Fishman, G.J., Grindlay, J.E., Barret, D., Tavani, M., Kaaret, P., Bloser, P., Ford, E., \& Titarchuk, L. 1996, A\&AS, 120C, 279

Zhang, S.N. 1997, in: D.T. Wickramasinghe, G.V. Bicknell \& L. Ferrario (eds.), Accretion Phenomena and Related Outflows, Proc. IAU Coll. No. 163, ASP-CS, 121, 41

Zhang, S.N., Cui, W., Harmon, B.A., Paciesas, W.S., Remillard, R.E., \& van Paradijs, J. 1997, ApJ (Letters), 477, L95

Zhang, S.N., Cui, W., \& Chen, W. 1997, ApJ (Letters), 482, L155

Zhang, S.N., Mirabel, I.F., Harmon, B.A., Kroeger, R.A., Rodriguez, L.F., Hjellming, R.M., \& Rupen, M.P. 1997, in: C.D. Dermer, M.S. Strickman \& J.D. Kurfess (eds.), Proc. Fourth Compton Symposium, AIP-CP, 410, 141

Zhang, S.N., Cui, W., Chen, W., Yao, Y., Zhang, X., Sun, X., Wu, X.-B., \& Xu, H. 2000, Science, 287,1239

Zhang, S.N. 2005, ApJ (Letters), 618, L79

Zhang, Y.-Z., Wang, J.-X., Attrill, G., \& Harra, L.K. 2006, in: V. Bothmer \& A.A. Hady (eds.), Solar Activity and its Magnetic Origin, Proc. IAU Symposium No. 233 (Cambridge: CUP), p. 357 